\title{
Dielectric response of electric - field distortions of the twist-bend nematic phase for LC Dimers
}

\author{
K. Merkel ${ }^{\S 1}$, C. Welch 2 , Z. Ahmed ${ }^{2}$, W. Piecek ${ }^{3}$, G.H. Mehl ${ }^{2}$ \\ ${ }^{1}$ Faculty of Computer Science and Material Science, Institute of Technology and \\ Mechatronics, University of Silesia in Katowice. Poland \\ ${ }^{2}$ Department of Chemistry, University of Hull, Hull HU6 7RX, UK \\ ${ }^{3}$ Faculty of Advanced Technologies and Chemistry, Military University of Technology, \\ Warszawa, Poland \\ ${ }^{\S}$ corresponding author: katarzyna.merkel@us.edu.pl
}

\begin{abstract}
Wide band dielectric spectroscopy of bent-shaped achiral liquid-crystal dimers 1 "'-n"-bis(4cyanobiphenyl-4'-yl) n-alkanes $(\mathrm{CBnCB} n=7,9,11)$ have been investigated in a frequency range $0.1 \mathrm{~Hz}$ to $100 \mathrm{MHz}$ using planar-aligned cells of sample thicknesses ranging from 2 to $10(\mu \mathrm{m})$ over a temperature range that covers both nematic and twist bend nematic phases. Two peaks in the dielectric spectrum in the higher frequency range are assigned to the molecular relaxation processes. The peak at the highest frequency, $\sim 40$ to $80 \mathrm{MHz}$, is assigned to an internal precessional rotation of a single unit of the dimer around the director. The mode in the next lower frequency range of 2 to $10 \mathrm{MHz}$ is assigned to the spinning rotation of the dimer around its long axis. This involves fluctuations of the dipole moment of the bent-shaped conformation that is directed along its arrow direction of the bow shape formed by the dimer. The peak in the frequency range $100 \mathrm{kHz}$ to $1 \mathrm{MHz}$ can be assigned to the collective fluctuations of the local director with reference to the helical axis of the $\mathrm{N}_{T B}$ structure. The dependence of its frequency on temperature is reminiscent of the soft mode observed at the $\mathrm{SmA}^{*}$ to $\mathrm{SmC}^{*}$ phase transition. This result clearly corresponds to the electroclinic effect - the response of the director to the applied electric field in an electro-optic experiment. The lowest frequency mode, observed in frequency range of $0.1 \mathrm{~Hz}-100 \mathrm{~Hz}$, is identified with the Goldstone mode. This mode is concerned with the long range azimuthal angle fluctuations of the local director. This leads to an alternating compression and expansion of the periodic structure of the $\mathrm{N}_{\mathrm{TB}}$ phase.
\end{abstract}




\section{Introduction}

There is an increasing interest of the soft matter community in the liquid crystals dimer research, mainly due to the occurrence of the first order nematic-nematic transitions and the interest in the observation of the materials properties of new nematic phases. Such properties include observation of stripes and nanoscale helical modulation with an oblique helicoidal angle. The interesting results arise from the (i) extraordinary flexoelectric $[1,2,3]$ and electro-optical properties $[4,5,6]$ of dimers and (ii) due to their ability of forming a modulated nematic $\left(\mathrm{N}_{\mathrm{TB}}\right)$ phase $[7,8,9,10,11,12,13]$. These dimers contain typically two rigid terminal groups which are chemically linked to each other by a flexible spacer of an odd number of methylene units $[14,15]$. The terminal groups can be different or the same and these lead to the two broad classes of LC dimers: asymmetric and symmetric, respectively. Recently, a combination of bent-core mesogens with calamitic units, have been shown to exhibit these properties $[16,17,18]$. The liquid crystalline dimers, as flexible molecules are likely to exhibit biaxial conformations and coupling between the conformational and the orientational distribution stabilizes the biaxial ordering. With the appearance of molecular bending, the bent elastic constant is significantly reduced and the molecular conformations can stabilize the new nematic phase $[17,19]$. This finally leads to the spontaneous formation of bent deformations of the director and an emergence of the new nematic order, in which the director follows an oblique helicoid by maintaining a constant angle with the helical axis [20, 21, 22]. This novel modulated nematic bulk phase, currently called the twist-bend nematic $\left(\mathrm{N}_{\mathrm{TB}}\right)$ forms a structural link in between the well-known uniaxial nematic $(\mathrm{N})$ and the chiral nematic $\left(\mathrm{N}^{*}\right)$ phases. In the $\mathrm{N}_{\mathrm{TB}}$ phase, the director exhibits periodic twist and bend deformations and these form a conical helix with an emergence of doubly degenerate domains of opposite handedness. The $\mathrm{N}_{\mathrm{TB}}$ phase in dimers, has experimentally been observed using a combination of experimental techniques such as the polarized light microscopy, X-ray diffraction (XRD) $[5,8,10,23,24]$, light scattering [9,24], freeze-fracture transmission electron microscopy (FFTEM) [5,8,100]. Additionally, the polarity and the chirality of the $\mathrm{N}_{\mathrm{TB}}$ phase formed by achiral molecules have been investigated by electro-optical [4,5,6,Error! Bookmark not defined.,11,17], nuclear magnetic resonance (NMR) [12, 23, 23, 25, 26, 27, 28, 29], EPR [30], magneto-optical [31], dielectric [17,18,18, 23, 27, 32] and Raman spectroscopy $[33,34]$. The most recent reports on $\mathrm{N}_{\mathrm{TB}}$ focus on to the study of the nanoscale pseudo-layered helical structures of the $\mathrm{N}_{\mathrm{TB}}$ phase using Resonant X-Ray Scattering (RXS) $[35,36,37]$. In addition, the theoretical models have attempted to describe 
the new nematic ground state with an anomalously low or a negative bend elastic constant $[38,39,40,41]$. These investigative methods suggest that a local helical structure is formed with an extremely small helical pitch of dimensions, $8-15 \mathrm{~nm}$. Such a helical modulation of the molecular orientation

at a nanoscale level leads to an extremely short response time of the director to the electric field. The time response of the electro-optic switching reported for the bent-core systems is of the order of $1 \mu \mathrm{s}$ [18]. It is even faster for CB7CB and CB11CB dimers and it lies in the submicrosecond range $[3,4,6]$ as compared to the millisecond range for uniaxial nematics. The study of the field-induced distortional effects in the $\mathrm{N}_{\text {TВ }}$ phase may be extremely useful for technological applications due to an analogy of the observed response with the electro-clinic effect $[4,42,43,44,45,46]$ of SmA. In the $\mathrm{N}_{\mathrm{TB}}$ phase, the effect of the electric field can be observed through both strong dielectric as well as weak flexoelectric couplings [47, 48, 49]. A linear electro-optic effect is observed where the optical axis is tilted by the field applied in a plane perpendicular to the helical axis. Such a characteristic effect resembles the electro-clinic effect, ECE, in the SmA* [3] and flexoelectric effect (FEE) $[42,48]$ in the $\mathrm{N}^{*}$. The analogy of the $\mathrm{N}_{\text {TB }}$ phase to the $\mathrm{N}^{*}$ is direct, since the properties of both phases arise from flexoelectricity due to the distortions of helical structure produced by the electric field. An analogy with the ECE in $\mathrm{SmA}^{*}$ can be understood in terms of a generalized analogy between the pseudo-lamellar $\mathrm{N}_{\mathrm{TB}}$ and the lamellar $\mathrm{SmA}^{*}$ [48]

This paper reports on our investigations of the molecular orientational dynamics of the $\mathrm{N}_{\text {TB }}$ mesophase formed by polar symmetric dimers CBnCB where $\mathrm{n}=9$ and 11 by studying the dielectric response with the DC field superimposed on a weak AC probe field in order to understand the interesting properties of such materials qualitatively and quantitatively better. Due to the formation of a periodic structure in the $\mathrm{N}_{\text {тв }}$ phase, the electro-optical response is similar to that of the smectic and cholesteric phases. On examining earlier reports on the CBnCB bimesogens [17,23,29,Error! Bookmark not defined.], we notice that observation of the soft and Goldstone modes have so far not been reported.

\section{Experimental Section}

\subsection{Description of the Compound}

The chemical structure of liquid-crystal dimers 1"-n"'-bis(4-cyanobiphenyl-4'-yl) n-alkanes $(\mathrm{CBnCB})$ is given in Figure 1 [50]; details of synthesis and surface properties for these materials have been reported recently [51]. Wide band dielectric spectroscopic study on 
planar-aligned cells is made in the frequency range of $0.1 \mathrm{~Hz}$ to $100 \mathrm{MHz}$ in the presence and absence of the DC bias fields using-impedance/network analysers: HP4I92A, HP4I95A and Solartron SI 1260. The cell-spacing is varied from 2 to $10 \mu \mathrm{m}$. These include planar commercial cells with SE-130 polymer aligning (Nissan Chemical Industries, Ltd). For higher frequency dielectric measurements, gold plated cells are filled with sample in their isotropic state. Dielectric measurements were performed on slow cooling $0.1 \mathrm{~K} / \mathrm{min}$. Temperature of the sample was stabilized using PID temperature controller within $\pm 2 \mathrm{mK}$. Pt100 platinum resistor was used as a sensor and 4 points method was applied to compensate the leads resistance. Sample was placed in the cooper block in order to reduce the temperature gradient across the sample.

The planar alignment in the range of the $\mathrm{N}$ and $\mathrm{N}_{T B}$ phases was controlled by observing the amplitude of high frequency mode. For thin samples, below $2 \mu \mathrm{m}$, almost uniform $\mathrm{N}_{\mathrm{TB}}$ texture, can be obtained by a slow cooling rate of $0.1 \mathrm{~K} / \mathrm{min}$, and further by growing domains and progressively merging, the stripes which organize into much larger bands as reported in [11]. The in-plane projection of the optic axis $\mathrm{N}$ of the $\mathrm{N}_{\text {TB }}$ phase is almost uniform, oriented approximately along the rubbing axis $\mathrm{Z}$, with a local deviation between $\mathrm{N}$ and $\mathrm{Z}$ of less than 2 deg. When confined in a $5 \mu \mathrm{m}$ cell we obtained well aligned strips and the rope texture can be removed by sample annealing or application triangular field. Insertion of a wave plate leads to different colours in adjacent stripes, indicating that the optical azimuthal angle alternates between stripes however the birefringence remain stable within the stripe, similarly as observed by Mandle and Goodby [52]. It seems the induced undulation preserves the coarse grain director to be in the plane of the substrate. The dielectric relaxation experiment for planar sample is sensitive only to vertical component of the electric permittivity, thus it does not depends on the planar undulation the coarse grain director. In the case of birefringence, we have measured it locally inside the strips, despite the coarse grain director varies in the plane across adjacent stripes.

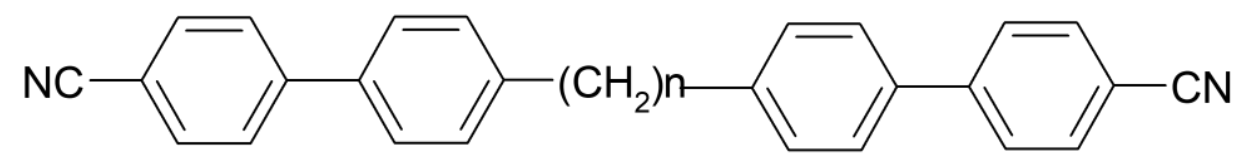

$\mathrm{n}=9,11$

CB9CB: $\quad \mathrm{Cr}-356.6 \mathrm{~K}-\mathrm{N}_{\mathrm{TB}}-380.1 \mathrm{~K}-\mathrm{N}-395.7 \mathrm{~K}-$ Iso

CB11CB: $\mathrm{Cr}-377.2 \mathrm{~K}-\mathrm{N}_{\mathrm{TB}}-381.9 \mathrm{~K}-\mathrm{N}-398.5 \mathrm{~K}-$ Iso

Fig.1. Chemical structure of $\mathrm{CBnCB}$ dimers and transition temperatures $[53,54,55]$. 


\subsection{Dielectric measurements}

We present dielectric results for planar $5 \mu \mathrm{m}$ sandwiched cells, the geometry of which is shown in the figure $2 \mathrm{a}$. The amplitude of the probe field is adjusted to lie in the range of $0.01-1 \mathrm{~V} / \mu \mathrm{m}$, where the dc bias fields up to $5 \mathrm{~V} / \mu \mathrm{m}$ are applied. The real $\left(\varepsilon^{\prime}\right)$ and imaginary $\left(\varepsilon^{\prime \prime}\right)$ parts of the complex permittivity are measured for the planar aligned cells. The measurements are made under slow cooling from the isotropic to nematic and $\mathrm{N}_{\text {тв }}$ phases. In order to determine the dielectric amplitude $\left(\delta \varepsilon_{\mathrm{j}}\right)$ and the relaxation time $\left(\tau_{\mathrm{j}}\right)$ of the $\mathrm{j}^{\text {th }}$ relaxation mode, the dielectric spectra are analyzed using Cole-Cole eq. (1) that expresses complex permittivity in terms of the parameters of the various relaxation processes.

$$
\varepsilon^{*}-\varepsilon_{\infty}=\sum_{j=1}^{n} \frac{\delta \varepsilon_{j}}{1+\left(i \omega \tau_{j}\right)^{\alpha_{j}}}
$$

Here, $\delta \varepsilon_{j}, \tau_{j}, \alpha_{j}, \varepsilon_{\infty}$ are the parameters of the $\mathrm{j}^{\mathrm{t}}$ relaxation process. These have been obtained from the fitting of the equation (1) to experimental data of $\varepsilon^{\prime \prime}$ or to the derivative of $\varepsilon^{\prime}$ with $\log (\mathrm{f}), n$ is the number of relaxation processes varying up to 4 .

Results of the fitting show the high frequency spectra are more complicated than displayed by a system that is just composed of rigid rod molecules. The $\mathrm{CBnCB}$ dimers are highly flexible, thus several conformers can contribute to the relaxation processes. The contributions to the dielectric spectra can arise from different rotations of components of the permanent molecular dipole moments. Contributions from the plausible conformational changes to the $\varepsilon$ " spectra from constituent parts of the molecule are being given. In the temperature range of the $\mathrm{N}_{\text {TB }}$ phase and for frequencies above $0.1 \mathrm{MHz}$, up to 4 relaxation peaks $(n \leq 4)$ are analysed. For obtaining a better deconvolution of the relaxation spectra in the frequency range above $0.1 \mathrm{MHz}$, it is preferable to analyze the derivative of the real part of permittivity $\varepsilon^{\prime}[56]$ with respect to $\ln f$, as given below:

$$
\frac{d \varepsilon^{\prime}}{d(\ln f)}=\frac{d \varepsilon^{\prime}}{d(\ln \omega)}=\sum_{j=1}^{n} \operatorname{Re} \frac{\delta \varepsilon_{j} \alpha_{j}\left(i \omega \tau_{j}\right)^{\alpha_{j}}}{\left[1+\left(i \omega \tau_{j}\right)^{\alpha_{j}}\right]^{2}}
$$

We choose the derivative method in order to reduce overlapping the modes in the spectra. Relaxation peaks, corresponding of each mode are symmetrical so the derivative of eps' vs $\log (\mathrm{f})$ makes/creates the peaks at the same frequency of maximum as for eps" but narrower, by factor 1.5 than the latter one (half width of the derivative is 0.756 (in the log scale) as compare to 1.144 for eps". 


\subsection{Birefringence measurements}

Birefringence measurements were performed using a commercial in-plane switching cell (IPS) with planar alignment parallel to the electrodes (Fig. 2b). The IPS cell coated by SE-130 polymer has a cell gap of $5 \mu \mathrm{m}(\mathrm{d}=4.91 \mu \mathrm{m})$ and $40 \mu \mathrm{m}$ gap between electrodes. The experiment was carried out by applying an $\mathrm{AC}$ electric field, that was $\mathrm{E}=5 \mathrm{~V} / \mu \mathrm{m}$ and experiments without external fields were performed as well. The optical properties of the $\mathrm{CBnCB}$ samples were investigated in the nematic and the twist-bend phases by polarizing microscope (Olympus BX56) equipped with Berek tilting Compensator (0-4 $\lambda$; U-CBE, Leica) and a digital camera. During measurements, a filter for wavelengths (546 nm) was used. We applied a $0.5 \mathrm{~Hz}$ AC field up to $10 \mathrm{~V} / \mu \mathrm{m}$ in the plane of the cell, perpendicular to the rubbing direction. The geometry of birefringence measurements and the orientation of the thin IPS cell in the laboratory frame xyz is shown in Figure $2 b$.

(a)

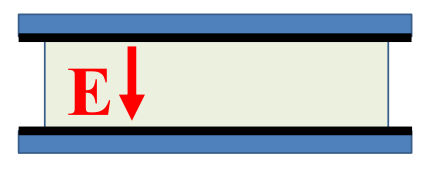

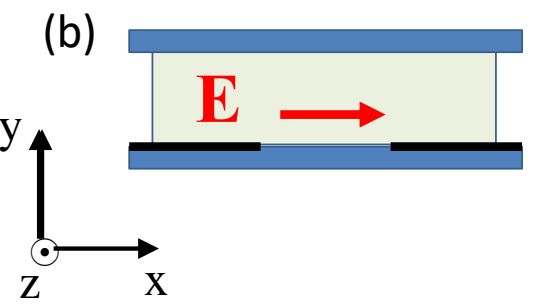

Fig.2. The schematics of the geometry of cells for the two alignments are shown: (a) planar sandwich cell. (b) planar in plane switching cell (IPS). Nematic director can be along X or Z axis.

\section{Results and discussion}

\subsection{Dielectric properties}

Figure 3 shows the fitting examples of the derivative of the real part of permittivity $\left(\varepsilon^{\prime}\right)$ with respect to $\log _{10} f\left(d \varepsilon^{\prime} / d \log _{10} f\right)$ at various temperatures $(378 \mathrm{~K}, 380 \mathrm{~K})$ and show too how the fittings reproduce the real $\left(\varepsilon^{\prime}\right)$ and imaginary part ( $\left.\varepsilon^{\prime \prime}\right)$ of the electric permittivity. Fitting with equation (2) to the derivative of the real electrical permeability, was first made for the lowest-frequency mode $\left(1 \mathrm{~Hz}-10^{2} \mathrm{~Hz}, \mathbf{m} 4\right)$ separately because its maximum is well isolated and does not coincide with others. Then, the parameters for mode $\mathbf{m} \mathbf{4}$ were fixed and used to fit the other three maxima of relaxation modes in higher frequency range. This procedure allowed us to limit the number of fitting parameters in the second step. From results of the 
fitting of the derivatives of the real part of the electric permittivity $\left(\varepsilon^{\prime}\right)$ to $\log _{10}(f)$, Eq. (2), three modes $\mathbf{m} 1, \mathbf{m} \mathbf{2}$ and $\mathbf{m} \mathbf{3}$ are found to exist above a frequency of $0.1 \mathrm{MHz}$. Figure 4 shows the 3-dm plot (dm-dimensional) of the derivative of the real part of the electric permittivity $\left(\varepsilon^{\prime}\right)$ vs. $\log _{10}$ of the frequency $\left(\mathrm{d} \varepsilon^{\prime} / \mathrm{dlog}_{10} \mathrm{f}\right)$ in the frequency range $1 \mathrm{kHz}-$ $100 \mathrm{MHz}$.

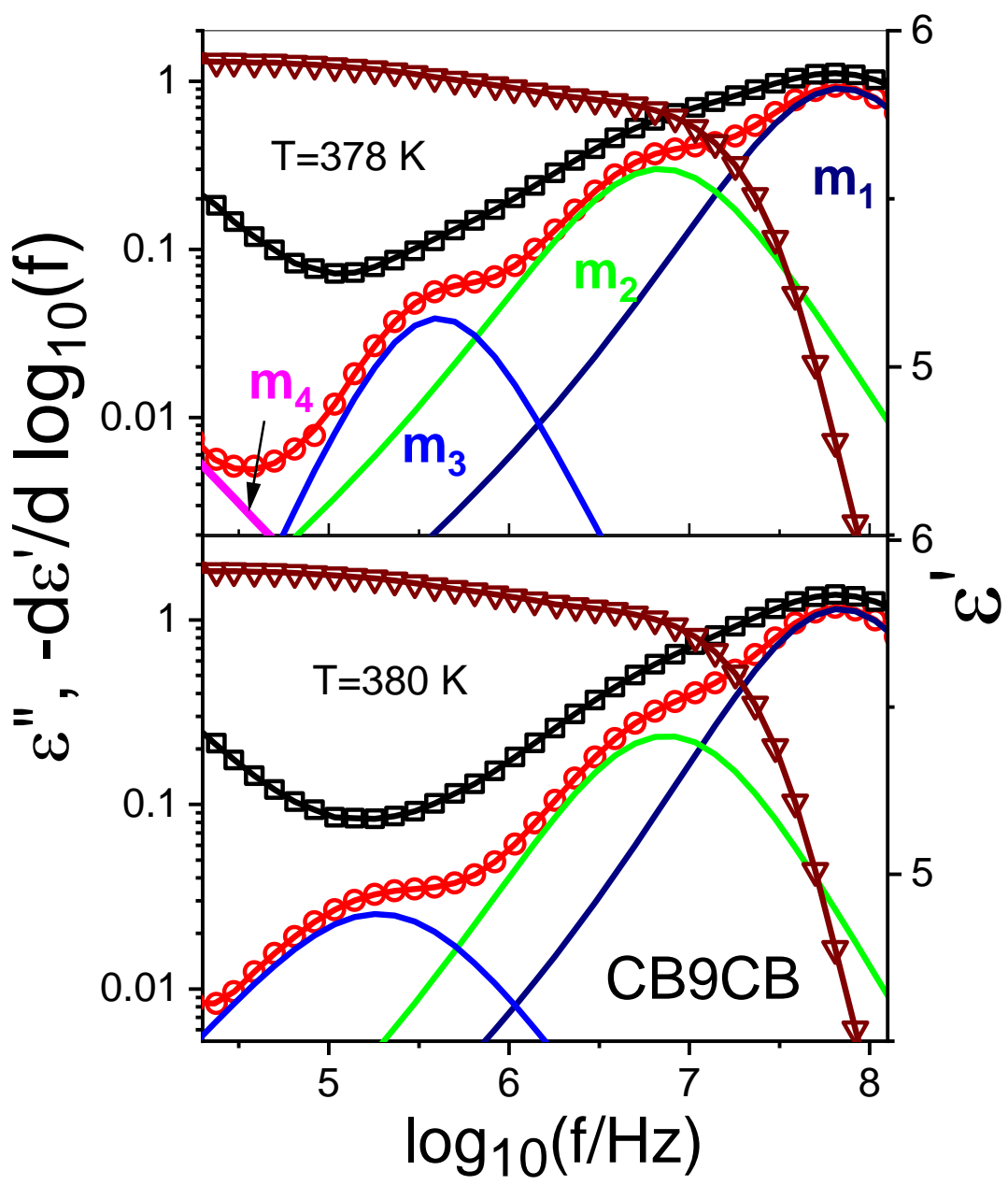

Fig.3. Frequency plots of the relative dielectric permittivity obtained for $5 \mu \mathrm{m}$ planar cell at different temperatures with respect to $\left(\log _{10} \mathrm{f}\right)$. Symbols represent experimental points for: $-\nabla$ - real part $\left(\varepsilon^{\prime}\right),-\square$ - imaginary part $\left(\varepsilon^{\prime \prime}\right)$ and -O- the derivative of $\varepsilon^{\prime}$. Solid lines are reproduced from fitting parameters. The navy, green, blue and magenta solid lines are the deconvoluted components of $\mathrm{d} \varepsilon^{\prime} / \mathrm{d}\left(\log _{10} f\right)$ and correspond to the contributing modes: $\mathbf{m}_{1}, \mathbf{m}_{2}$, m3, m4 . 


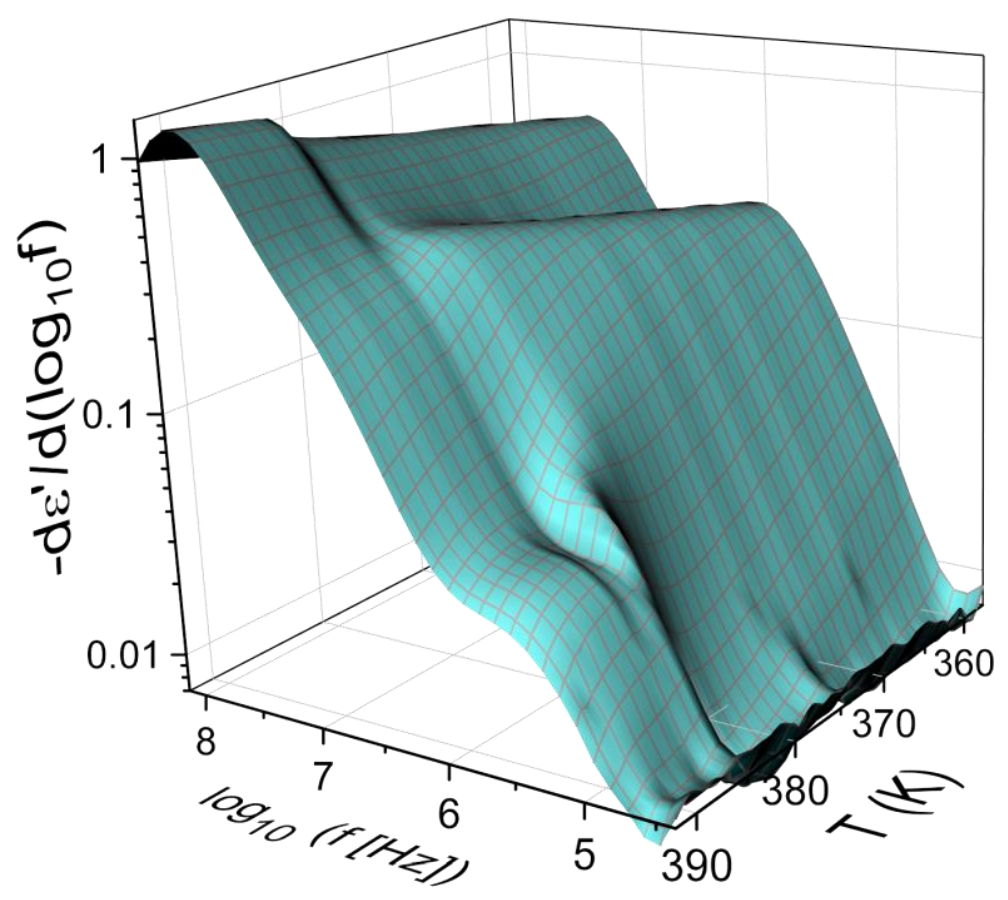

Fig.4. 3-D plot of the derivative of the real permittivity $\left(\varepsilon^{\prime}\right)$ vs. $\log _{10}$ of frequency $\left(\mathrm{d} \varepsilon^{\prime} / \mathrm{d}\left(\log _{10} \mathrm{f}\right)\right)$ for $5 \mu \mathrm{m}$ planar cell of CB9CB sample as a function of the frequency and the temperature.

A set of these relaxation frequencies is plotted as a function of temperature in Figure 5. The corresponding relaxation frequency, $f_{\mathrm{j}}=1 / 2 \pi \tau_{\mathrm{j}}$, is calculated from $\tau_{\mathrm{j}}$. The $\alpha_{\mathrm{j}}$ for each mode is found to be negligibly small as it lies in a restricted range of values of $0.05-0.1$. The temperature dependencies of the dielectric amplitudes of these modes are plotted in Figure 6. For a temperature range of the uniaxial $\mathrm{N}$ phase, the spectra are found to be even more complex. An additional peak $\mathbf{m} \mathbf{2}^{\prime}$ is observed from deconvolution of the fittings. This peak is due to the existence of other conformers that possibly may exist at a given temperature in the $\mathrm{N}$ phase. The mode $\mathbf{m} \mathbf{2}^{\prime}$ is assigned to contributions from the hairpin-shaped conformers to the dielectric spectra as was reported by Lopez et al [32] and it dominates in the $\mathrm{N}$ phase due to its large dipole moment as compared to other conformers. The low frequency peak (below $1 \mathrm{kHz}$ ) is analyzed separately since it is stand alone and does not overlap with other peaks.

The Maier and Meier (M-M) static model was extended qualitatively to dynamics by Toriyama et al [57]. However a set of detailed quantitative calculations for the rotational diffusion model were carried out by Coffey and Kalmykov $[58,18]$ in which they derived the equation for the perpendicular and parallel component of the complex dielectric permittivity.

For nematic dimers dielectric relaxation can be discussed in the framework of Stocchero 
et al.'s theoretical model [59]. A major difference for is that for dimers there are transitions amongst four stable states instead of two. Main conclusion that can be drawn is that: while the behaviour of the parallel component significantly depends on the molecular structure the perpendicular component of the permittivity does not influence the temperature dependence on it [60]. In the paper we analysed only the perpendicular component of the permittivity $\varepsilon_{\perp}^{*}(\omega)$ thus we can use calculations for the rotational diffusion model, given below:

$$
\varepsilon_{\perp}^{*}(\omega)-\varepsilon_{\perp \infty} \approx \frac{N^{\prime} g h F^{2}}{3 \varepsilon_{0} k_{B} T}\left[\frac{\mu_{l}^{2}(1-S)}{1+i \omega \tau_{10}}+\frac{\mu_{t}^{2}\left(1+\frac{1}{2} S\right)}{1+i \omega \tau_{11}}\right]
$$

Here, $A=N^{\prime} g h F^{2} / 3 \varepsilon_{0} k_{B}$ is the scaling factor of the two relaxation processes that contribute to the complex permittivity. $N^{\prime}(=M / d)$ is the number density of molecules, $d$ is the mass density, $M$ is the molecular weight, $\varepsilon_{0}$ is the permittivity of vacuum, $T$ is the absolute temperature, $k_{B}$ is the Boltzmann constant, $g$ is the Kirkwood correlation factor introduced to account for dipole-dipole correlations. The Kirkwood correlation factor is usually assumed to be unity in the $N$ phase. $F$ and $h$ are the internal field factors for the reaction and cavity fields, respectively. Dipole components: $\mu_{\mathrm{l}}$ and $\mu_{\mathrm{t}}$ are the longitudinal and the transverse components, respectively, of the total molecular dipole moment $(\mu)$. Dipole components $(\mu$ I and $\mu_{\mathrm{t}}$ ) are directed normal to the long molecular axis. Thus the two terms on the right-hand side of Eq. (3) relax at different frequencies given by $f_{\mathrm{j}}=1 / 2 \pi \tau_{\mathrm{j}}$, depending on the relaxation time $\tau_{\mathrm{j}}$ of the $\mathrm{j}^{\text {th }}$ mode. Here $\tau_{10}$ and $\tau_{11}$ are the relaxation times for the precessional and the spinning rotations of the dipole components (longitudinal and the transverse, respectively). The expressions for $\tau_{10}$ and $\tau_{11}$ in terms of relaxation time of the isotropic state $\left(\tau_{0}\right)$ involve other two parameters, the orientational order parameter $(S)$ and the anisotropy in the rotational diffusion coefficients $(\Delta)$. The latter is given by $\Delta=\frac{1}{2}\left[D_{\|} / D_{\perp}-1\right], D_{\|}$and $D_{\perp}$ are the parallel and the normal components of the rotational diffusion coefficients, respectively. The expressions for $\tau_{10}$ and $\tau_{11}$ are given as:

$$
\begin{aligned}
& \frac{\tau_{10}}{\tau_{0}}=\frac{1-S}{1+\frac{1}{2} S} \\
& \frac{\tau_{11}}{\tau_{0}}=\frac{2+S}{2+\Delta(2+S)-\frac{1}{2} S}
\end{aligned}
$$




\subsection{Molecular modes}

As already stated, the $\varepsilon^{\prime \prime}$ spectra at higher frequencies are dominated by two peaks. These correspond to the two molecular relaxation modes $\mathbf{m} \mathbf{1}$ and $\mathbf{m} \mathbf{2}$ of the $\mathrm{CBnCB}$ dimer, as also made by Cestari et al [23] and López et al [32]. The relaxation peaks of the CB9CB dimer at a temperature of $379 \mathrm{~K}$ are found from fitting at frequencies of $f_{\mathrm{m} 1}=60 \mathrm{MHz}$ and $f_{\mathrm{m} 2}=5 \mathrm{MHz}$, corresponding to $\mu_{\mathrm{t}}$ and $\mu_{\mathrm{l}}$ components of the permanent dipole moment, respectively. The dielectric amplitudes and their relaxation frequencies for each of these processes are plotted as a function of temperature in Figure 5. The numerical results agree with those reported earlier for the $N_{\text {TB }}$ phase [27,32]. However, a third peak corresponding to mode $\mathbf{m} \mathbf{3}$ is additionally found by the deconvolution of the relaxation spectra; frequency of this mode is centered at $f_{\mathrm{m} 3}=1 \mathrm{MHz}$. An emergence of this dielectric peak has not been reported previously in the literature. In order to carefully analyze the third peak in the presence of the other two we have to make the fitting of all three of them using eq. (2).

Theoretical models of flexible dimers suggest that there are two main populations of conformers, extended bent core and hairpin-like. The population of extended conformers is growing upon cooling [23] In the $\mathrm{N}_{\mathrm{TB}}$, the contributions to the dielectric spectra of the bentshaped conformers are significant/dominant. In the $\mathrm{N}_{\mathrm{TB}}$, the contributions to the dielectric spectra of the bent-shaped conformers are significant. As dimers under investigations are symmetric, the longitudinal component of the dipole moment, in their most extended linear configuration, is zero. Following equation (3), the contribution to the perpendicular component of permittivity originates from the spinning rotation of the transverse component of the dipole moment i.e. rotation of the bow axis of the bent-core conformation contributes significantly to the perpendicular component. This is the assignment of mode $\mathbf{m} \mathbf{2}$. The higher frequency relaxation process, $\mathbf{m} \mathbf{1}$, can arise from the rotations of a segment of the molecule, i.e. the internal rotation of each monomer with the spacer anchored, involves the fluctuations of the cyanobiphenyl dipolar moment. As the length of the spacer in between the two mesogenes in the dimer is large enough such an independent internal rotation of each monomer of the dimer is highly feasible. The temperature dependencies of $\delta \varepsilon_{\mathrm{t}}$ and of the relaxation time $\tau_{10}$ are suggestive of the precessional rotation of the longitudinal component of the each cyanobiphenyl dipole moment around the director in a planar-aligned cell. If this mode were to originate from the precessional motion of the entire dipole moment of the dimer, the relaxation rate would have to be much lower than that for $\mathbf{m} \mathbf{2}$, contrary to experimental observations. Though numerical results for modes $\mathbf{m} \mathbf{1}$ and $\mathbf{m} \mathbf{2}$ for the CB9CB 
are similar to those of Cestari et al. [23], nevertheless the mechanism of assignments are different than those given earlier in the literature.

Results in the $\mathrm{N}$ phase are quite complicated as the dielectric amplitude and its relaxation rate are strongly affected by the reorganization of conformers in terms of molecular shapes. Two major conformers exist: the bent core conformer with a transversal dipole moment and a hairpin-shaped conformer with a large longitudinal dipole moment. This is clearly reflected in the results of the temperature dependencies of the dielectric amplitude $\delta \varepsilon_{2}$ of the $\mathbf{m} \mathbf{2}$ mode. At the phase transition from the $\mathrm{N}$ to the $\mathrm{N}_{\text {Тв }}$ phase, the dielectric amplitude of $\mathbf{m} \mathbf{2}$ increases significantly due to an increase in the population density of the bent conformers, see Fig. 6 for the CB9CB. We can see differences in the relaxation times of the mode $\mathbf{m} \mathbf{2}$ and those reported by M. Cestari et al [23] and D. O. López et al [32] as they obtained them for $\mathbf{m} \mathbf{2}^{\prime}$ mode for homeotropic aligned cells.

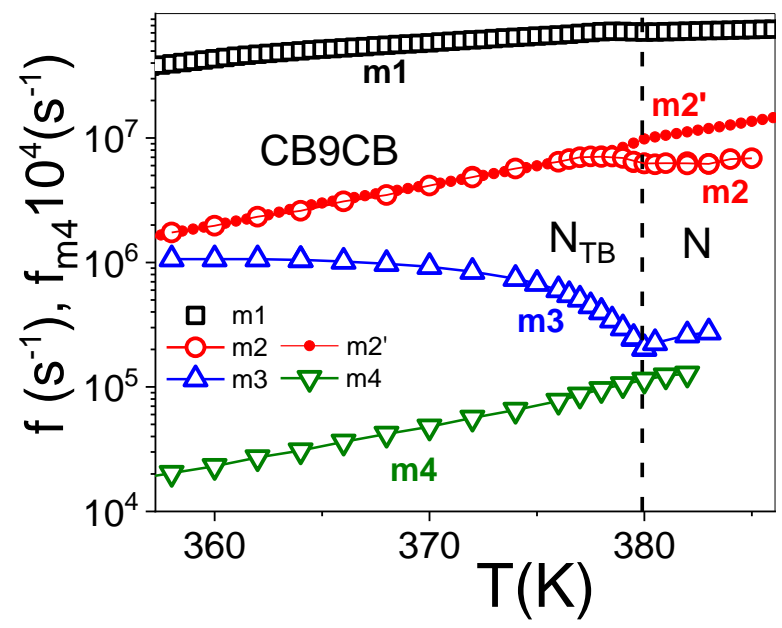

Fig. 5. Plots of the relaxation frequencies as a function of temperature, for CB9CB dimer. Our results for modes: $\mathbf{m} 1-\square-, \mathbf{m} 2-\mathrm{O}_{-}, \mathbf{m} 3-\triangle$ - and $\mathbf{m} \mathbf{4}-\nabla-$. The $\mathbf{m} \mathbf{1}$ mode is the rotation of the monomer involving $\boldsymbol{\mu}$; describes by the relaxation time, $\boldsymbol{\tau} \mathbf{1 0}$. The $\mathbf{m} \mathbf{2}$ is the spinning rotation of the dimer dipole and involves $\boldsymbol{\mu}_{t}$. Mode $\mathbf{m} \mathbf{2}^{\prime}$ reported by López et al [32] -0-, is redrawn for comparison. Frequency of mode $\mathbf{m} \mathbf{4}$ is multiplied by $10^{4}$. The $\mathbf{m} \mathbf{4}$ is a mode at the lowest range of frequencies, $1 \mathrm{~Hz}$ to $100 \mathrm{~Hz}$, used in these investigations.

They achieved homeotropic alignment by applying a strong electric field across the electrodes of the cell. The external field may increase the population of hairpin-shaped conformers in the $N$ phase as they possess large longitudinal dipole moments and these will therefore significantly affect the dielectric parameters. These dimers display different dynamics than those given for the bent core conformers in the cell arrangements we use. 


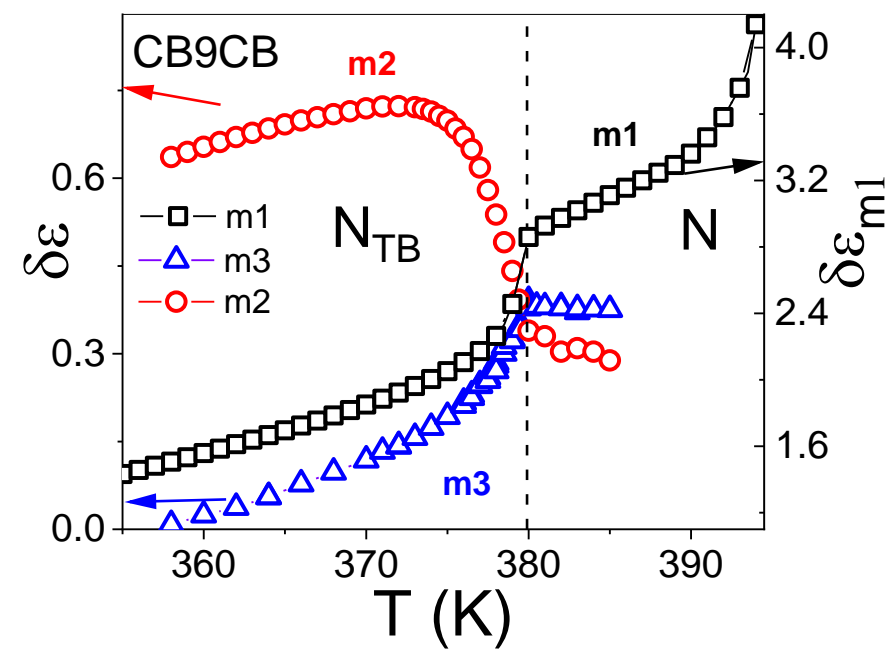

Fig. 6. Plots of the the dielectric amplitudes $\delta \varepsilon$, for modes: $\mathbf{m} 1-\square-, \mathbf{m} 2-\mathrm{O}_{-}, \mathbf{m} 3-\triangle$-. The dielectric amplitude for mode $\mathbf{m} 3$, is multiplied by a factor of 4 .

\subsection{Order parameter and the cone angle}

The dielectric amplitudes and the relaxation frequencies of molecular modes in general yield useful information about the molecular dynamics of the dipolar system investigated. The characteristics of these modes in terms of peak frequencies and dielectric amplitudes are used to calculate the order parameter $(S)$ in the $\mathrm{N}$ and the $\mathrm{N}_{\text {TB }}$ phases. The dielectric amplitude of the $\mathbf{m} \mathbf{2}$ mode is clearly influenced by the changes that occur in the shape of conformers, these also increase complications in the analysis of the data; especially in the $\mathrm{N}$ phase. The dipole moment of the biphenyl group lies along the monomer thus the relaxation rate of the $\mathbf{m} \mathbf{1}$ is an excellent probe for finding $S$. We can obtain $S$ by carrying out a fitting of the dielectric amplitude $\delta \varepsilon_{1}$ of the mode $\mathbf{m} \mathbf{1}$ in the isotropic and the $\mathrm{N}$ phases as follows:

$$
\delta \varepsilon_{C B}=(1-S) \mu_{l}^{2} N^{\prime} h F^{2} / 3 \varepsilon_{0} k_{B} T
$$

where: $\mu_{l}^{2} h F^{2} / 3 \varepsilon_{0} k_{B}$ is assumed to be a constant of fixed value for both nematic and isotropic phases. The results for the obtained $S$ parameter for CB9CB are shown in Figure 7a. They can be well fitted with the eq.:

$$
S=\left(1-T / T^{*}\right)^{b},
$$

Where: $T^{*}=396.5 \mathrm{~K}$ corresponding to the $\mathrm{N}-\mathrm{I}$ phase transition temperature and $b$ is critical exponent, $b=0.21$. 
(a)

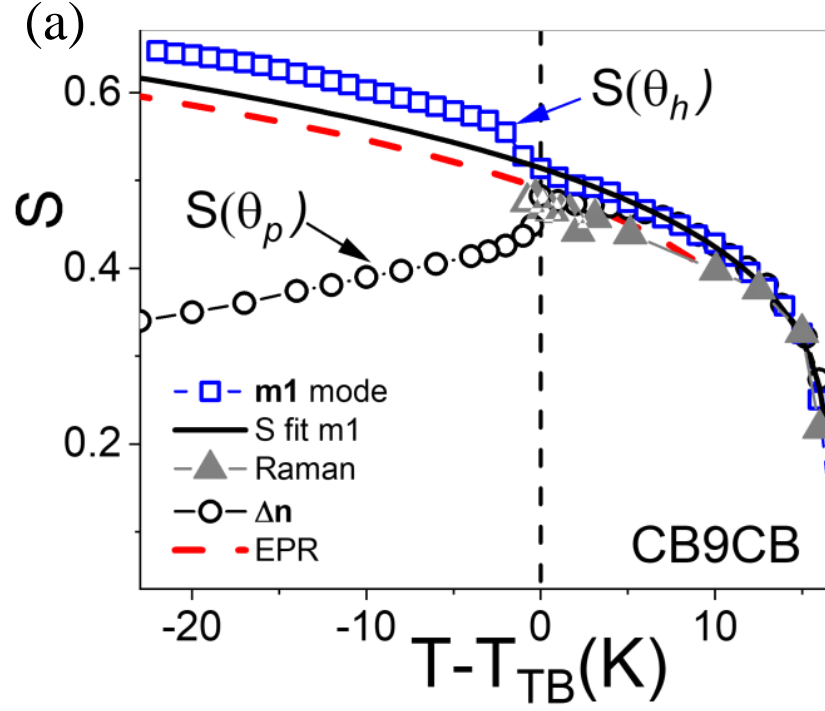

(b)

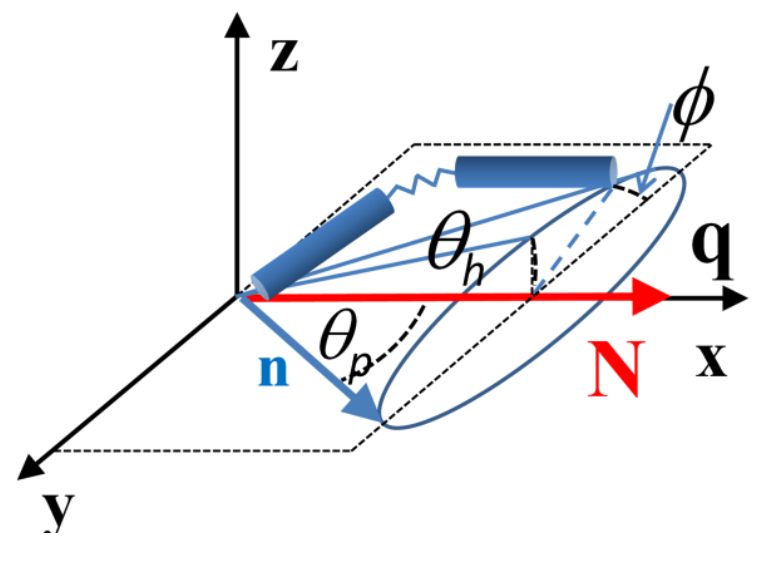

Fig. 7. (a) The orientational order parameter $(S)$ for CB9CB obtained from the dielectric amplitude $\left(\delta \varepsilon_{1}\right)$ of mode $\mathbf{m 1}$ of monomer (precessional mode of the cyanobiphenyl dipole) plotted as a function of the reduced temperature, $-\square$ - and its extrapolation due to eq.(6) shown by black solid line, - $S$ calculated from $\Delta \boldsymbol{n}$ of planar aligned sample. Literature $S$ data redrawn for comparative study: --- EPR [30], - $\Delta$ - Raman spectroscopy [Error! Bookmark not defined.]. (b) Scheme showing the biaxial distribution of the local director $\boldsymbol{n}$ in the planar sample. $N$ is a coarse-grained director, $\theta_{p}$ and $\theta_{h}$ are a measure of the director distribution in the substrate plane and across the cell, respectively, and $q$ is the wave vector.

Another method, useful for probing the orientational order is birefringence measurement. In the $\mathrm{N}$ phase, the results are very similar to those from dielectric experiments, if one excludes results close to the $\mathrm{N}-\mathrm{N}_{\mathrm{TB}}$ transition temperature $(\mathrm{T}<385 \mathrm{~K})$. These results can be well fitted using the classical Haller formula:

$$
\Delta n=\Delta n_{0}\left(1-T / T^{*}\right)^{b}
$$

Result of the fitting is given by parameters: $\Delta n=0.314, b=0.186$ and $T^{*}=397.2 \mathrm{~K}$, corresponding to the $\mathrm{N}-\mathrm{I}$ phase transition temperature. The extrapolation of the temperature dependence of the resulting $S$ parameter is shown by a black dash line in the Figure 8 . Both dielectric and birefringence results are found to be in good agreement with those obtained by Raman and EPR spectroscopies reported in the literature [33,Error! Bookmark not defined.] as shown in the Figure 7a.

In the $\mathrm{N}_{\mathrm{TB}}$ phase both methods show a quite different behavior of the orientational order. It is worth noticing that the dielectric method is probing the dipole distribution across the cell (i.e. with respect to the substrate plane- $\left.\left\langle\sin ^{2}\left(\theta_{h}\right)\right\rangle\right)$ because the electric field is perpendicular 
to substrate, however in the birefringence method the field of the light beam is in the plane of substrate; so this method is probing the induced dipole distribution in the plane of the cell $\left\langle\sin ^{2}\left(\theta_{p}\right)\right\rangle$. The angles for the director distribution can be defined as: $\theta_{p}$ is a measure the director distribution in the substrate plane and $\theta_{h}$ is a measure of the director distribution across the cell (out of the plane) (Fig.7b). In the $\mathrm{N}_{\text {TB }}$ phase the apparent order parameter $\left(S_{\mathrm{p}}\right)$ from the birefringence method (solid black circles in Fig.7a) is described by the following eq.:

$$
S_{p}=S P_{2}\left(\cos \theta_{p}\right)
$$

where: $P_{2}\left(\cos \theta_{p}\right)$ is the Legendre polynomial $\left.P_{2}\left(\cos \theta_{p}\right)=0.5\left(3 \cos ^{2} \theta_{p}-1\right)\right)$, (black solid line in Figure 8).

The resulting angle; $\theta_{p}$, is approaching $27^{\circ}$ at $30 \mathrm{~K}$ below the transition to the $\mathrm{N}_{\text {TB }}$ phase and its dependence on $T$ is shown in Fig. 8. The $\left\langle\sin ^{2}\left(\theta_{p}\right)\right\rangle$ obtained from birefringence data shows the distribution of the local director in the plane of substrate (Fig. 7b). On the other hand the apparent value of the order parameter from the dielectric method clearly exceeds the value extrapolated from it's the N phase (black solid line). Surprisingly, the distribution of the local director looks confined more tightly to the substrate plane than in the $\mathrm{N}$ phase. Thus the distribution of the local director is appearing biaxial, $\left\langle\sin ^{2}\left(\theta_{h}\right)\right\rangle\left\langle\left\langle\sin ^{2}\left(\theta_{p}\right)\right\rangle\right.$ (see Fig. 7b). We can evaluate $\left\langle\sin ^{2}\left(\theta_{h}\right)\right\rangle$ directly from the definition of the order parameter $S=1-3 / 2\left\langle\sin ^{2}\left(\theta_{h}\right)\right\rangle$, seen by dielectric method. The director is initially tilted about $18^{\circ}$ out of the substrate plane on entering the $\mathrm{N}_{\text {TB }}$ phase and gradually its orientation is becoming more planar, when $S$ is approaching 0.7 at about $20 \mathrm{~K}$ below the transition temperature. It is worth noting that the dipole moments of the cynaobiphenyl groups make an angle $\beta$ (of about $26^{\circ}$ ) with respect to the bow axis of the dimer. This is close to the values detected for structurally related dimers using GIXRD and RSXD methods [36]. Thus any further increase of the $S$ parameter for the CB dipole is only possible provided the bow arrow dipole of the dimer aligns with the substrate plane. 

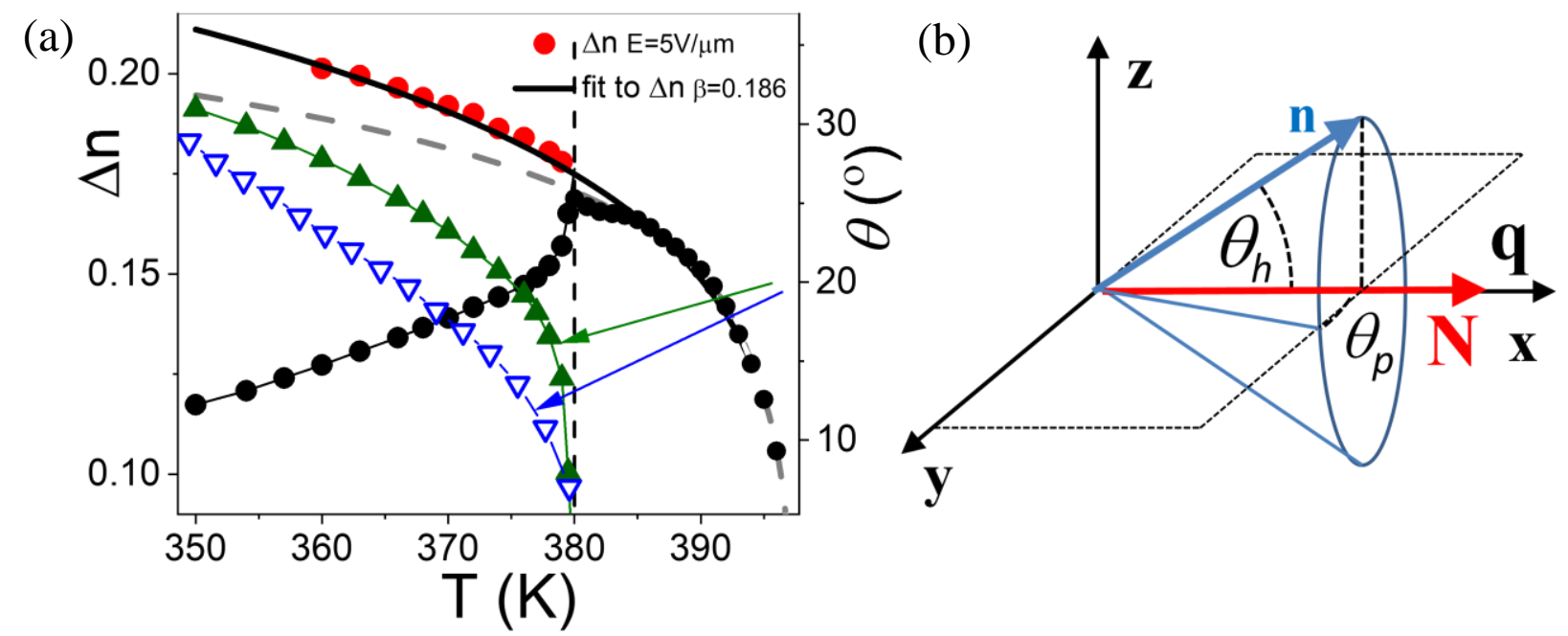

Fig. 8. Temperature dependence of the birefringence $\Delta n,-\mathbf{- E}-\mathrm{off}, \bigcirc, \mathrm{E}=5 \mathrm{~V} / \mu \mathrm{m}$, and the heliconical angle in the $\mathrm{N}_{\mathrm{TB}}$ phase of $\mathrm{CB} 9 \mathrm{CB}$, calculated from the E-off birefringence measurements in the planar sample, black solid line - is an extrapolation to dielectric data as in Fig.7a, gray dash line - is an extrapolation to the birefringence $\Delta n$. - $\mathbf{\Delta}$ - Literature NMR data redrawn for comparative study [52,53]: $-\triangle$-. (b) Scheme showing the biaxial distribution of the local director $\boldsymbol{n}$ in the planar sample after applying the field $E=5 \mathrm{~V} / \mu \mathrm{m}$.

On applying a $\mathrm{AC}$ field, $\mathrm{E}=5 \mathrm{~V} / \mu \mathrm{m}$, we observe an increase of the order within $\mathrm{z}$-x plane, $\triangle n$ is becoming larger then extrapolated from the $\mathrm{N}$ phase, $\triangle n>\triangle n_{\mathrm{N}}$, thus indicating the increase of the orientational order in z-x plane. If we combine these findings with the results on the $\mathbf{m} \mathbf{1}$ mode we can conclude that the field effect can simply be interpreted as a rotation of a biaxiality plane from $x-y$ to $x-z$ plane.

Similar procedure has been applied for CB11CB sample in order to obtain orientational order parameter. Figure 9 shows the order parameter calculated from dielectric $\mathbf{m} \mathbf{1}$ mode in the similar way as for CB9CB. In $\mathrm{N}_{\mathrm{TB}}$ phase orientational order becomes lower than expected from that extrapolated dependence. Using eq. 8 the cone angle in NTB phase is obtained. The angle is growing up $13^{\circ}$ and then saturates at $377 \mathrm{~K}$ due to glass transition. The results do not exclude other similar organizations. In particular the possibility of the conic angle not being unique, but rather of varying with the molecular segments of the dimers has been recently put forward by Vanakaras and Photinos [61] and could provide a possible molecular origin of the tilt distribution. A discussion about a possible more complex structure of the twist-bend phase of $\mathrm{CB} 11 \mathrm{CB}$ has also been recently provided by Mandle et al. [62], while yet another possibility, recently put forward, is that the axis of the helix is not fixed but undulates. 


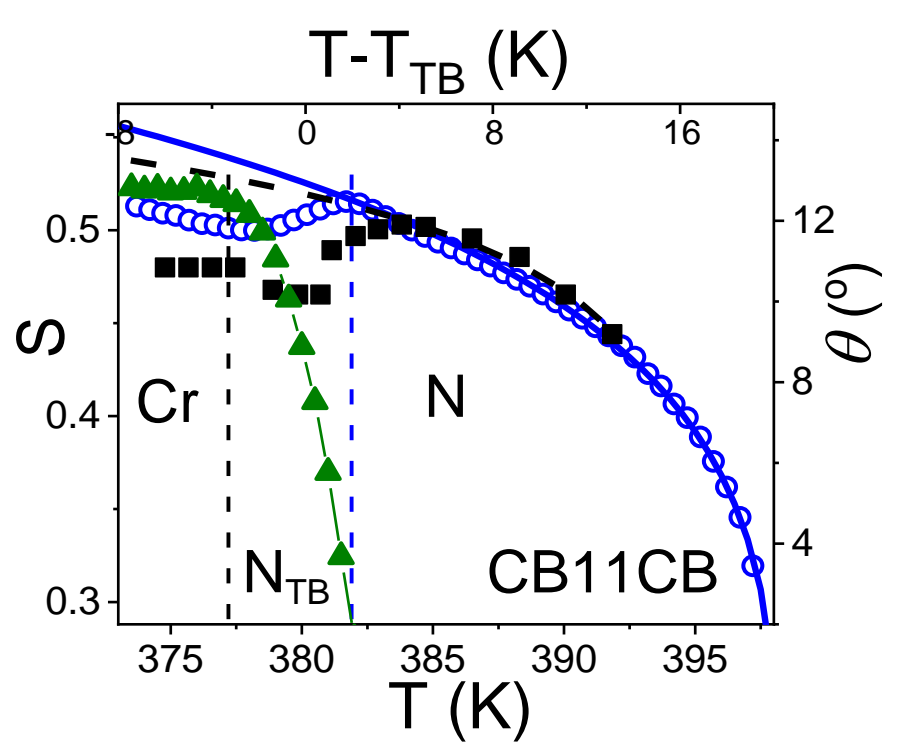

Fig. 9. (a) The orientational order parameter $(S)$ for $\mathrm{CB} 11 \mathrm{CB}$ obtained from the dielectric amplitude $\left(\delta \varepsilon_{1}\right)$ of mode $\mathbf{m} \mathbf{1}$ plotted as a function of the temperature, - $\mathrm{O}_{-}$, its extrapolation due to eq.(6) shown by blue solid line, and the heliconical angle in the $\mathrm{N}_{\mathrm{TB}}$ phase of CB11CB, calculated from the dielectric data: $-\Delta_{-}^{-}$. Literature $S$ from EPR data [54] redrawn for comparative study and its extrapolation due to eq.(6) shown by black broken line.

This can be well reproduced by eq. 6 with the fitting parameters: $b=0.21$ and $T^{*}=398.6 \mathrm{~K}$. Results are in good accordance with literature $S$ data from EPR [54] and Raman spectroscopy [33].

Our data cannot clearly distinguish between these different models at this stage, but we hope that this work will stimulate further analysis of these phases.

\subsection{Orientational viscosity coefficient}

The dynamical behavior of mode $\mathbf{m} 2$ reveals that its relaxation time, denoted by $\tau_{11}$, is dependent on the order parameter $(S)$ and the anisotropy in the diffusion coefficients $(\Delta)$. We can reproduce the relaxation time $\tau_{11}$ satisfactorily by using the $S$ parameter obtained as described above. As a result, the anisotropy in the diffusion coefficients $(\Delta)$ can be obtained, and this increases from 0.4 to 0.9 on approaching the transition from the $\mathrm{N}$ to the $\mathrm{N}_{\text {TB }}$ phase, similar to the results reported by Cifelli et al [29] for CB7CB- $\mathrm{d}_{4}$.

The temperature dependence of the anisotropy in the diffusion coefficients $(\Delta)$ clearly shows that the spinning rotation accelerates at the transition from the $\mathrm{N}$ to the $\mathrm{N}_{T B}$ phase, similar to the case CB7CB (Fig. 10). 


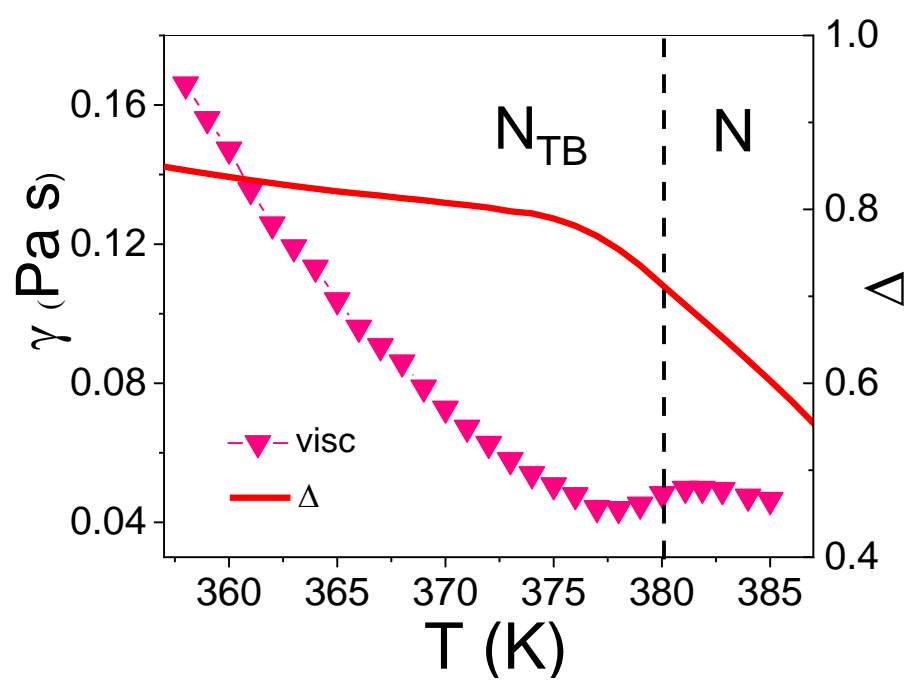

Fig. 10. Plots of the viscosity coefficient, $\gamma,-\nabla$ - and anisotropy of diffusion coefficient: $\Delta$ red solid line, calculated from the relaxation time of $\mathbf{m} 2$ mode.

The relaxation time of mode $\mathbf{m} \mathbf{2}$, on the other hand, can be related to the rotational viscosity $(\gamma)$ using the formula:

$$
\tau_{11}=3 \gamma_{1} V \lambda_{11} / k_{B} T
$$

$V$ is the volume per molecule of a LC dimer and $\lambda$ is a Perrin friction factor of an asymmetric ellipsoid's spinning rotation about the longest axis [63]. The factor, $\lambda$, was calculated to be 0.3 and 0.28 for dimers: $\mathrm{CB} 9 \mathrm{CB}$ and $\mathrm{CB} 11 \mathrm{CB}$ respectively. These are approximated by an ellipsoid with the ratio of the lengths of axes: 1:0.32:0.24 and 1:0.30:0.23 for CB9CB and CB11CB, respectively. Thus the dynamic parameters for the coefficients of viscosity and diffusion indicate the acceleration of the rotation of the precession motion in the phase transition from the $\mathrm{N}$ to the $\mathrm{N}_{\text {TB }}$ phase. The results for the viscosity coefficients are plotted at Figure 10, and will be used for simulation of the dynamic of collective modes.

\subsection{Collective modes}

The dielectric peak corresponding to the $\mathbf{m} \mathbf{3}$ mode shows a quite intriguing behavior with regards to the temperature dependence of the frequency and its amplitude at the $\mathrm{N}_{T B}$ to the $\mathrm{N}$ transition (see Fig. 5). This result is reminiscent of the behaviour of a soft mode at the transition from the $\mathrm{SmA}^{*}$ to the $\mathrm{SmC}^{*}$ phase. The dielectric peak can be assigned to the collective fluctuations of the tilt angle which the coarse-grained director $N$ makes with the normal of the pseudo-layers. This effect is similar to that reported for an asymmetric bentcore molecule reported recently by some of us [18]. One can expect that the electro-optic 
effects in the $\mathrm{N}_{\mathrm{TB}}$ phase are similar to the in-plane flexoelectric switching in the $\mathrm{N}^{*}$ phase. Such an electro-optic phenomenon was observed for the CB11CB dimer by Panov et al. [4,7] and for the CB7CB dimer by Meyer et al [64]. More extensive theoretical and experimental studies by Meyer et al. [6] showed that this effect is also similar to the electro-clinic effect (ECE) in the SmA* phase. The $\mathrm{N}_{\mathrm{TB}}$ and the cholesteric phases have several common characteristic features. Both phases are periodic in the pseudo-layered structures and both of these systems are chiral. The cholesteric phase arises from the molecular chirality, whereas the twist-bend nematic arises from the structural chirality of the heliconical structure. Therefore, in all the three phases: $\mathrm{N}^{*}, \mathrm{SmA}^{*}$ and $\mathrm{N}_{\mathrm{TB}}$, when an electric field $\mathbf{E}$ is applied in a plane of the layers (or pseudo-layers), the optic axis is tilted from its initial orientation to a new position in a plane at right angles to the electric field. In other words in a planar aligned cell, the director shifts in a plane parallel to the electrodes when the field is applied across the cell. Such an effect is only possible in chiral systems, thus this observation confirms that the $\mathrm{N}_{\text {TB }}$ phase possesses chiral symmetry even if it is formed by achiral molecules Panov [4]. The distortions in the director occur by the field and the relaxation time of the corresponding mode is written as $[6,9]$ :

$$
\Gamma=1 / \tau_{t}=2 \pi f_{t}=\frac{q^{2} K_{t} \sin ^{2} \theta}{\gamma}
$$

where: $K_{\mathrm{t}}=\left(K_{1}+K_{2}\right) / 2$ is the effective elastic constant for the tilt of the coarse-grained director $N$ shifted from its pseudo-layer normal position to a new position under the field, $q$ is the wave vector. When the high frequency AC probe field is applied, we can neglect a variation in the wave vector $q(=2 \pi / p)$, induced by the probe field, ie. we assume that the pitch $p$ of the helical structure is dynamically frozen at its field-off value [6]. The rotational viscosity $(\gamma)$ is expected to be continuous at the transition temperature from the $\mathrm{N}$ to the $\mathrm{N}_{\mathrm{TB}}$

We can try to reproduce the temperature dependencies of the relaxation rate by using experimentally available parameters for the $\mathrm{CBnCB}$ dimers based on eq. (10). We use the viscosity obtained from the $\mathbf{m} \mathbf{2}$ mode (see Fig. 10). Values of the helical pitch of the $N_{\mathrm{TB}}$ and thus wavenumber $q$ are obtained from the data on the resonant carbon K-edge soft X-ray scattering made by Zhu et al [35] for the CB7CB dimer. These are rescaled by the length ratio of the dimers (d-CBnCB/d-CB7CB). 


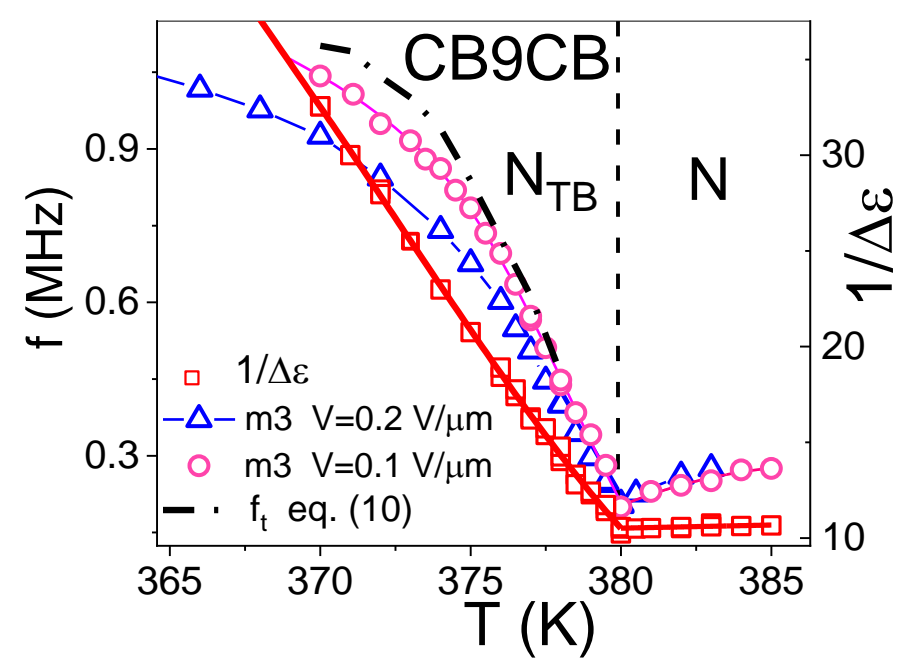

Fig. 11. The relaxation frequency of the tilt deformation $\mathbf{m} 3$ mode for CB9CB dimer: -O$\mathrm{E}=0.1 \mathrm{~V} / \mu \mathrm{m},-\triangle-\mathrm{E}=0.2 \mathrm{~V} / \mu \mathrm{m},-\square$ - reciprocal value of $\mathbf{m 3}$ amplitude $(1 / \Delta \varepsilon)$ and its linear fit (red dash line),. -..-- (black dash dot line) - the soft mode frequency $f_{\mathrm{t}}$ due to eq. (10) $\left(K_{\mathrm{t}} \cong\right.$ $9 \mathrm{pN})$.

The temperature dependence of the cone angle is calculated from birefringence measurements. Finally the predicted temperature dependence of the soft mode frequency due to eq. (10) $\left(\mathrm{K}_{\mathrm{t}} \cong 9 \mathrm{pN}\right.$ was estimated as reported by Robles-Hernández et al [27]) is calculated and shown in the Figure 5. This only slightly exceeds the experimental value as can be expected for a non-disturbed heliconical structure. The resulting temperature dependence of the mode frequency shows specific softening behaviour on approaching the transition temperature from the $\mathrm{N}$ to the $\mathrm{N}_{\text {TB }}$ phase.

We can compare the above results with the relaxation rate calculated from the electrooptic experiment carried out by Meyer et al [63]. The electro-optic data show initially a slight increase in the relaxation rate on decreasing the temperature, this tendency reverses below $465 \mathrm{~K}$. But overall the dependence on temperature is intriguing and it shows a much slower relaxation rate at $(\sim 0.4 \mathrm{MHz})$ than found from our dielectric relaxation data. It is also interesting to find the reason of such a discrepancy (of one order of magnitude). In fact the electro-optic experiment by C. Meyer et al was carried out at reasonably large amplitude of the electric field $25 \mathrm{~V} / \mu \mathrm{m}$. Nonetheless Meyer et al showed that as the response was sufficiently fast (10 $\mu \mathrm{s})$, the heliconical structure was conserved.

We can determine the strength of the influence of the field on the dielectric response. Figure 11 and 12 shows the results of two probe fields: $0.1 \mathrm{~V} / \mu \mathrm{m}$ and $0.2 \mathrm{~V} / \mu \mathrm{m}$; with increasing the amplitude of the field the field dependence gradually becomes less steep. 


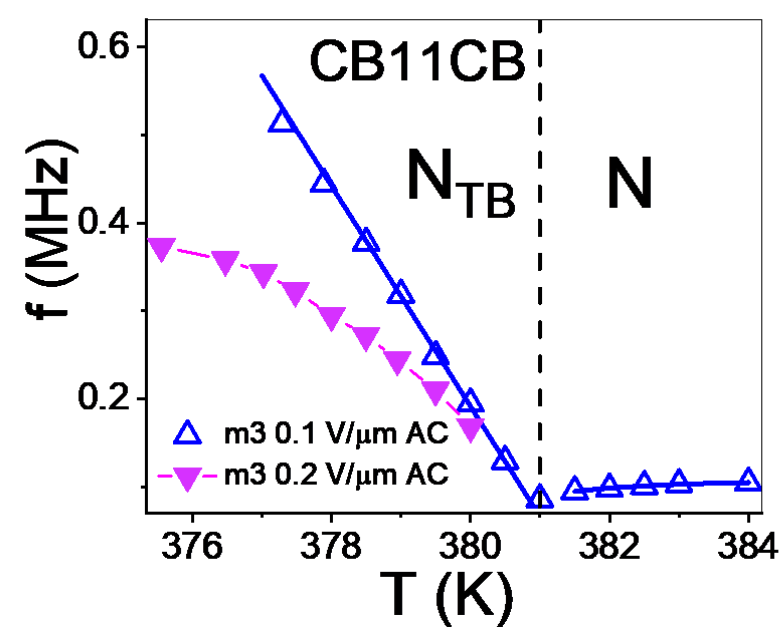

Fig. 12. Relaxation frequency of the tilt deformation $\mathbf{m} 3$ mode for CB11CB molecule: $-\triangle$ $\mathrm{E}=0.1 \mathrm{~V} / \mu \mathrm{m} \mathrm{AC},-\nabla-\mathrm{E}=0.2 \mathrm{~V} / \mu \mathrm{m} \mathrm{AC}$.

This would indicate the deformation/rearrangement of the heliconical structure, i.e. reducing the wavenumber of the helix has occurred. The corresponding $\mathrm{f} \sim q^{2}$ decreases by $10 \%$ and $20 \%$ relatively to the unperturbed case for electric fields of $0.1 \mathrm{~V} / \mu \mathrm{m}$ and $0.2 \mathrm{~V} / \mu \mathrm{m}$, respectively. Thus, it is quite clear that the field applied in the reported electro-optic experiment can significantly deform the heliconical structure. The corresponding value for the switching time can be estimated to increase more than two times with respect to the field-off helical structure.

The lowest frequency relaxation process, $\mathbf{m} \mathbf{4}$, is observed in the frequency range $1 \mathrm{~Hz}-$ $10^{2} \mathrm{~Hz}$. This can be identified with a Goldstone mode, related to long-scale fluctuations of the azimuthal angle of the local director $\boldsymbol{n}$, which does not change the coarse-grained director, $\boldsymbol{N}$ [Error! Bookmark not defined.,18,38]. Such a long wavelength fluctuation mode should be analogous to those observed for the smectic-A phase, in particular the "slow", hydrodynamic layer compression-bending mode (or "undulation" mode). This mode (m4) may have a few other possibilities that can be considered as an origin of the mode. At the low frequency range of the spectra the flexoelectric instability and electro-convection in planar nematic cell are possible. This effect can be ruled up as it can only be observed above some threshold field and its contribution usually develops on increasing the field. The existence of the low frequency mode has been already reported in the nematic phase of bent shape molecules both by light scattering [65] and dielectric relaxation [66]. Relative to straight cores, close packing of orientationally ordered bent-shape molecules would tend to favour smectic layering on short length scales. The surface area of the clusters (so-called smectic "cybotactic" clusters) would be significantly greater than for a single molecule, leading to larger rotational 
viscosities and correspondingly slower orientational fluctuations. Thus we are expected a continuous increase in mean cluster size on decreasing temperature or on increasing electric field, which are, however, not observed in the experiment. Another concept of the mode origin is based on a dynamic light scattering study [65] of a bent-core nematic phase. By exploiting the polarization selection rules of the scattering, it is possible to detected separate modes attributable to uniaxial director fluctuations and, in the lower part of the nematic phase, to fluctuations of the biaxial order parameter. The striking fact is that the relaxation rate of the director modes in the bent-core material is roughly two orders of magnitude lower than the corresponding rate observed in typical straight-core nematics This is quite similar concept as offered in present paper except we insist that fluctuation are due to long range azimuthal director fluctuation.

Figure 13a shows the frequency dependence of the imaginary part of dielectric permittivity $\left(\varepsilon^{\prime \prime}\right)$ for a planar-aligned cell displayed at different AC voltage.

(a)

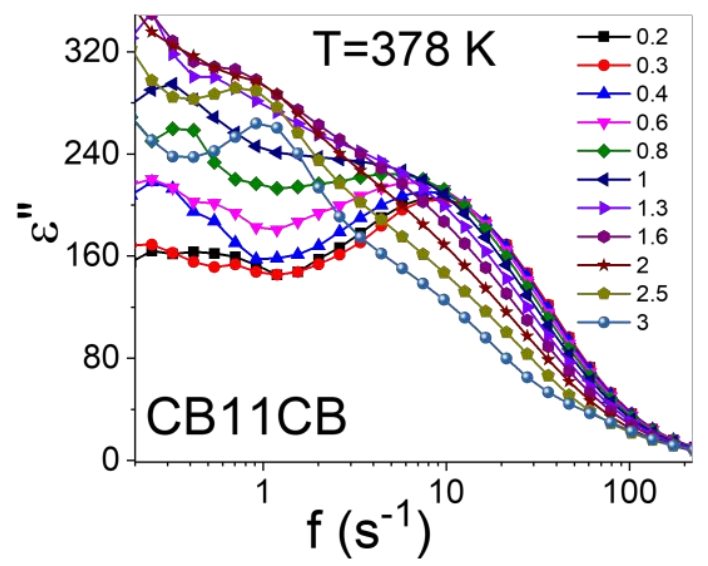

(b)

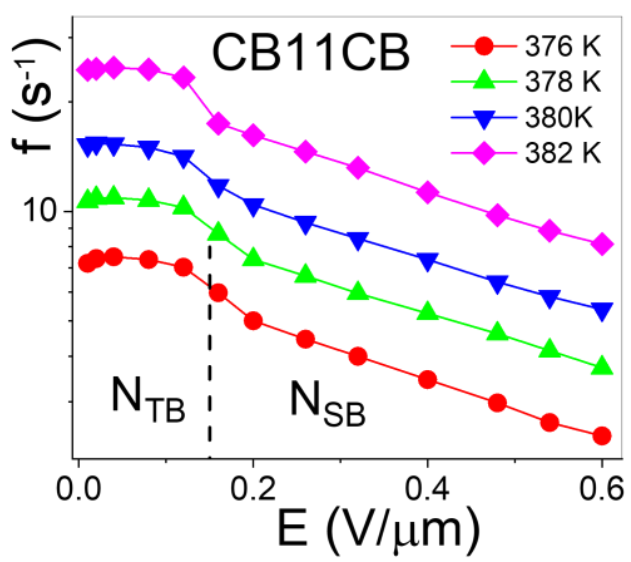

Fig.13. (a): The $\varepsilon^{\prime \prime}$ spectra for a planar-aligned cell displayed at different AC voltage $(5 \mu \mathrm{m}$ cell), (b) Frequency of the low frequency mode $f \sim q^{2}$ plotted as a function of $E$, for temperatures: $376 \mathrm{~K}-0-, 378 \mathrm{~K}-\mathbf{\Delta}-, 380 \mathrm{~K}-\nabla$ - and $382 \mathrm{~K}-\bullet-$.

This mode combines "pseudo-layer" compression and bending, which should to be described by an effective layer compression elastic constant, $B_{\text {eff }}$ [9]:

$$
\Gamma_{S}=2 \pi f_{S}=\frac{B_{e f f}}{\gamma}
$$

where the effective elasticity $B_{\text {eff }}$ associated with $K_{2}$ and $K_{3}$, plays the role of the elastic moduli for compression of the pseudo-layer structure in the $\mathrm{N}_{\mathrm{TB}}$ phase and $\gamma$ is the relevant viscosity coefficient, $\left(B_{\text {eff }} \sim K_{3} q^{2} \sin ^{2} \theta\right)$ [63]. The low frequency dielectric results confirms 
the presence of the "pseudo-layer" structure of the $\mathrm{N}_{\mathrm{TB}}$ phase with exceptionally low $B_{\text {eff }}$ being below $10^{4} \mathrm{~Pa}$. The magnitude of $B_{\text {eff }}$ differs substantially from the typical value of $B_{\text {eff }}$ $=10^{6} \mathrm{~Pa}$ in a conventional smectic-A.

The peak frequency of the mode shows quite interesting dependence with the increasing amplitude of the measuring field (Fig 13b). For small field amplitude, E $<0.03 \mathrm{~V} / \mu \mathrm{m}$ the peak frequency increases, then remain constant for $\mathrm{E}<0.15 \mathrm{~V} / \mu \mathrm{m}$ and suddenly drop at about $\mathrm{E}=0.2$ $\mathrm{V} / \mu \mathrm{m}$. After that, peak frequency decreases almost linearly on increasing field amplitude. It is shown [9] the relaxation rate of the mode can be related to the wavenumber of the heliconical structure, $f \sim q^{2}$, thus the field simply increase the pitch of the periodic structure. Similar field dependence of the mode frequency and related helical wavenumber has been observed for bent-core system showing $\mathrm{N}_{\mathrm{TB}}$ phase [18]. Such behaviour was well predicted by theoretical model Pająk et al [39], where the drop of frequency or wavenumber was interpreted as follows the transition from $\mathrm{N}_{\mathrm{TB}}$ to $\mathrm{N}_{\mathrm{SB}}$ structure.

It is intriguing, that the Goldstone mode survives in the lower end of the nematic phase, despite the non-chiral nature of the nematic phase. There is considerable progress in understanding the new dynamic mode of symmetry breaking, based on the synchronization of the chirality of temporally chiral molecules. This process leads to spontaneous deracemisation in the liquid state under thermodynamic control, giving rise to long-lasting stable symmetrybroken fluids, even at high temperatures. Chiral structures can arise from achiral building blocks that lack a handedness if their assembly is unstable to chiral distortions, a phenomenon called spontaneous symmetry breaking. Chirality in LC requires transiently chiral molecules capable of adopting chiral conformations or configurations and it leads to liquid conglomerates efficient in chirality amplification, and eventually to macroscopic homo-chiral mono-domains, developing either from weak chiral perturbations or from random fluctuations. First Link et al. [67] pointed out the chirality induced by tilting of BSMs. In the case of $\mathrm{CBnCB}(\mathrm{n}=7,5,11)$ dimers we clearly observe chirality in $\mathrm{N}_{\mathrm{TB}}$ phase, which manifest itself by heliconical structure. However, it is noticeable even deeply nematic phase that molecules are well tilted, which is indicated both by low order parameter ( $\mathrm{S}$ ranged 0.4-0.55) and unusual temperature dependence of the birefringence that does not follows known models for classical nematic phase. This is accompanied by strong director fluctuation in the nematic phase that might be responsible for spontaneous symmetry breaking and the induced chirality. It was recently shown evidence of strong fluctuation twist bent deformation of the nematic director deeply in nematic phase far above the $\mathrm{N}-\mathrm{N}_{\mathrm{TB}}$ transition [68]. This results in nonzero efficient value of the cone angle, which is usually observed by birefringence measurements. 


\section{Conclusions}

The dielectric spectra of the twist-bend nematic phase $\left(\mathrm{N}_{\mathrm{TB}}\right)$ of an achiral liquid crystalline dimer are investigated in the frequency range $0.1 \mathrm{~Hz}$ to $100 \mathrm{MHz}$ in order to determine its response to the electric field. We observe four modes in the dielectric spectra, the two higher frequency ones are assigned to the molecular modes and the other two are assigned to the collective modes that in turn are due to the distortion of the heliconical structure by the field. The collective mode in MHz region is assigned to the local distortions of the conical angle while the periodic helical structure remains unaltered. The temperature dependencies of the relaxation frequency* of each mode and of the resulting elastic modulus have anomalous softening-like behaviour at the $\mathrm{N}-\mathrm{N}_{\mathrm{TB}}$ transition. The lowest frequency collective mode, observed in the frequency range $1 \mathrm{~Hz}-10^{2} \mathrm{~Hz}$ is particularly interesting and is identified with the Goldstone mode, related to the long-scale fluctuations of the phase of the local director. This results in alternating compression and expansion of the pseudo-layered structure.

\section{Acknowledgement}

Author (KM) acknowledges funding through the National Science Centre, Poland for Grant No. 2018/31/B/ST3/03609. Author (KM) thanks Prof. J.K. Vij for fruitful discussions. CW and GHM acknowledge funding through the EPSRC grant EP/M015726/1.

\section{References}

[1] S.M. Morris, M.J. Clarke, A.E. Blatch, and H.J. Coles, "Structure-flexoelastic properties of bimesogenic liquid crystals" Phys. Rev. E, 75, 041701 (2007).

[2] S.M. Shamid, S. Dhakal, and J.V. Selinger, "Statistical mechanics of bend flexoelectricity and the twist-bend phase in bent-core liquid crystals" Phys. Rev. E, 87, 052503 (2013).

[3] C. Meyer, G.R. Luckhurst, and I. Dozov, "Flexoelectrically driven electroclinic effect in the twist-bend nematic phase of achiral molecules with bent shapes" Phys. Rev. Lett., 111, 067801 (2013).

[4] V.P. Panov, R. Balachandran, M. Nagaraj, J.K. Vij, M.G. Tamba, A. Kohlmeier, and G.H. Mehl, "Microsecond linear optical response in the unusual nematic phase of achiral bimesogens” Appl. Phys. Lett., 99, 261903 (2011).

[5] D. Chen, J.H. Porada, J.B. Hooper, A. Klittnick, Y. Shena, M.R. Tuchband, E. Korblova, D. Bedrov, D.M. Walba, M.A. Glaser, J.E. Maclennan, and N.A. Clarka, "Chiral heliconical ground state of nanoscale pitch in a nematic liquid crystal of achiral 
molecular dimers" Proc. Natl. Acad. Sci., 110, 15931 (2013).

[6] C. Meyer, "Nematic twist-bend phase under external constraints" Liq. Cryst., 43, 2144 (2016).

[7] a) V.P. Panov, M. Nagaraj, A. Kocot, Y. Panarin, J.K. Vij, G.H. Mehl, P-3.36, 23rd ILCC, Kraków (2010), b) V.P. Panov, M. Nagaraj, J.K. Vij, Yu.P. Panarin, A. Kohlmeier, M.G. Tamba, R.A. Lewis, and G.H. Mehl, Phys. Rev. Lett., 105, 167801, (2010).

[8] V. Borshch, Y.K. Kim, J. Xiang, M. Gao, A. Jakli, V.P. Panov, J.K. Vij, C.T. Imrie, M.G. Tamba, G.H. Mehl, and O.D. Lavrentovich, Nat. Comm., 4, 2635 (2013).

[9] Z Parsouzi, S.M. Shamid, V. Borshch, P.K. Challa, A.R. Baldwin, M.G. Tamba, C. Welch, G.H. Mehl, J.T. Gleeson, A. Jakli, O.D., Lavrentovich, D.W. Allender, J.V. Selinger and S. Sprunt, Phys. Rev. X, 6, 021041 (2016).

[10] D. Chen, M. Nakata, R. Shao, M.R. Tuchband, M. Shuai, U. Baumeister, W. Weissflog, D.M. Walba, M.A. Glaser, J.E. Maclennan, N.A. Clark, "Twist-bend heliconical chiral nematic liquid crystal phase of an achiral rigid bent-core mesogen" Phys. Rev. E 89, 022506 (2014).

[11] C. Meyer, G.R. Luckhurst, and I. Dozov, "The temperature dependence of the heliconical tilt angle in the twist-bend nematic phase of the odd dimer CB7CB" J. Mater. Chem. C, 3, 318 (2015).

[12] J.P. Jokisaari, G.R. Luckhurst, B.A. Timimi, J. Zhu, and H. Zimmermann, “Twist-bend nematic phase of the liquid crystal dimer CB7CB: orientational order and conical angle determined by ${ }^{129} \mathrm{Xe}$ and ${ }^{2} \mathrm{H}$ NMR spectroscopy" Liq. Cryst., 42, 708 (2015).

[13] D.A. Paterson, R. Walker, J.P. Abberley, J. Forestier, W.T.A. Harrison, J.M.D. Storey, D. Pociecha, E. Gorecka, C.T. Imrie, “Azobenzene-based liquid crystal dimers and the twist-bend nematic phase" Liq. Cryst., 44, 2060 (2017).

[14] C.T. Imrie and G.R. Luckhurst, "Liquid crystal dimers and oligomers" in Handbook of Liquid Crystals Vol. 2B: Low Molecular Weight Liquid Crystals, edited by D. Demus, J.W. Goodby, G.W. Gray, H.W. Spiess, and V. Vill (Wiley-VCH, Weinheim, Germany, 1998), Chap. X, p. 801, b) C.T. Imrie and P.A. Henderson, "Liquid crystal dimers and higher oligomers: Between monomers and polymers," Chem. Soc. Rev., 36, 2096 (2007).

[15] R.J. Mandle, "The dependency of twist-bend nematic liquid crystals on molecular structure: A progression from dimers to trimers, oligomers and polymers" Soft Matter, 
12, 7883 (2016).

[16] M.G. Tamba, B. Kosata, K. Pelz, S. Diele, G. Pelzl, Z. Vakhovskaya, H. Kresse, W. Weissflog, "Mesogenic dimers composed of a calamitic and a bent-core mesogenic unit" Soft Matter, 2, 60 (2006).

[17] R. Balachandran, V.P. Panov, J.K. Vij, G. Shanker, C. Tschierske, K. Merkel, A. Kocot, "Dielectric and electro-optic studies of a bimesogenic liquid crystal composed of bentcore and calamitic units" Phys. Rev. E, 90, 032506 (2014).

[18] K. Merkel, A. Kocot, J.K. Vij, and G. Shanker, "Distortions in structures of the twist bend nematic phase of a bent-core liquid crystal by the electric field" Phys. Rev. E, 98, 022704 (2018).

[19] N. Sebastian, M.G. Tamba, R. Stannarius, M.R. de la Fuente, M. Salamonczyk, G. Cukrov, J. Gleeson, S. Sprunt, A. Jakli, C. Welch, Z. Ahmed, G.H. Mehl, and A. Eremin, "Mesophase structure and behaviour in bulk and restricted geometry of a dimeric compound exhibiting a nematic-nematic transition" Phys. Chem. Chem. Phys., 18, 19299 (2016).

[20] R.B. Meyer. "Structural problems in liquid crystals physics. Les houches summer school in theoretical physics. In: Balian R, Weill G, editors. Molecular fluids. Vol. XXV1973. New York: Gordon and Breach. Les Houches lectures; 1976. p. 273-373.

[21] I. Dozov, "On the spontaneous symmetry breaking in the mesophases of achiral bananashaped molecules On the spontaneous symmetry breaking in the mesophases of achiral banana-shaped molecules" EurPhys. Lett., 56, 247 (2001).

[22] R. Memmer, "Liquid crystal phases of achiral banana shaped molecules: a computer simulation study" Liq Cryst. 29, 483 (2002).

[23] M. Cestari S. Diez-Berart, D.A. Dunmur, A. Ferrarini, M.R. de la Fuente, D.J.B. Jackson, D.O. Lopez, G.R. Luckhurst, M.A Perez-Jubindo, R.M. Richardson, J. Salud, B.A. Timimi, H. Zimmermann, "Phase behavior and properties of the liquid-crystal dimer 1",7"-bis(4-cyanobiphenyl-4'-yl) heptane: A twist-bend nematic liquid crystal" Phys. Rev. E, 84, 031704 (2011).

[24] K. Adlem M. Čopič, G.R. Luckhurst, A. Mertelj, O. Parri, R.M. Richardson, B.D. Snow, B.A. Timimi, R.P.Tuffin, D. Wilkes, "Chemically induced twist-bend nematic liquid crystals, liquid crystal dimers, and negative elastic constants" Phys. Rev. E, 88, 022503 (2013). 
[25] L. Beguin, J.W. Emsley, M. Lelli, A. Lesage, G.R. Luckhurst, B.A. Timimi, and H. Zimmermann, "The chirality of a twist-bend nematic phase identified by NMR spectroscopy" J. Phys. Chem. B, 116, 7940 (2012).

[26] J.W. Emsley, M. Lelli, A. Lesage, and G.R. Luckhurst, "A comparison of the conformational distributions of the achiral symmetric liquid crystal dimer CB7CB in the achiral nematic and chiral twist-bend nematic phases" J. Phys. Chem. B, 117, 6547 (2013).

[27] B. Robles-Hernández, N. Sebastián, M.R. de la Fuente, D.O. López, S. Diez-Berart, J. Salud, M.B. Ros, D.A. Dunmur, G.R. Luckhurst, B.A.Timimi, "Twist, tilt, and orientational order at the nematic to twist-bend nematic phase transition of 1",9"-bis(4cyanobiphenyl-4'-yl) nonane: A dielectric, H 2 NMR, and calorimetric study" Phys. Rev. E, 92, 062505 (2015).

[28] A. Hoffmann, A. G. Vanakaras, A. Kohlmeier, G. H. Mehl, and D. J. Photinos, "On the structure of the Nx phase of symmetric dimers: Inferences from NMR," Soft Matter, 11, $850(2015)$.

[29] M. Cifelli, V. Domenici, S.V. Dvinskikh, G.R. Luckhurst, and B.A. Timimi, "The twistbend nematic phase: translational self-diffusion and biaxiality studied by $1 \mathrm{H}$ nuclear magnetic resonance diffusometry" Liq. Cryst., 44, 204 (2017).

[30] C. Bacchiocchi, M.-G. Tamba, G.H. Mehl, A. Arcioni, I. Miglioli, and C. Zannoni, "EPR study of the polydomain structure of the twist-bend nematic phase of $\mathrm{CB} 9 \mathrm{CB}$ in the bulk" Liq. Cryst., 45,2109 (2018).

[31] S.M. Salili, J. Xiang, H. Wang, Q. Li, D.A. Paterson, J.M.D. Storey, C.T. Imrie, O.D. Lavrentovich, S.N. Sprunt, J.T. Gleeson, A. Jákli, "Magnetically tunable selective reflection of light by heliconical cholesterics” Phys. Rev. E, 94, 042705 (2016).

[32] D.O. López, N. Sebastian, M.R. de la Fuente, J.C. Martínez-García, J. Salud, M.A. Pérez-Jubindo, S. Diez-Berart, D.A. Dunmur, G.R. Luckhurst, "Disentangling molecular motions involved in the glass transition of a twist-bend nematic liquid crystal through dielectric studies" J. Chem. Phys., 137, 034502 (2012).

[33] Z. Zhang, V.P. Panov, M. Nagaraj, R.J. Mandle, J.W. Goodby, G.R. Luckhurst, J.C. Jonesad and H.F. Gleeson "Raman scattering studies of order parameters in liquid crystalline dimers exhibiting the nematic and twist-bend nematic phases" J. Mater. Chem. C, 3, 10007 (2015). 
[34] N. Trbojevic , D.J. Read, M. Nagaraj „Dielectric properties of liquid crystalline dimer mixtures exhibiting the nematic and twist-bend nematic phases" Phys. Rev. E, 96, 052703 (2017).

[35] C. Zhu, M.R. Tuchband, A. Young, M. Shuai, A. Scarbrough, D.M. Walba, J.E. Maclennan, Ch. Wang, A. Hexemer, N.A. Clark, "Resonant Carbon K -Edge Soft XRay Scattering from Lattice-Free Heliconical Molecular Ordering: Soft Dilative Elasticity of the Twist-Bend Liquid Crystal Phase" Phys. Rev. Lett., 116, 147803 (2016).

[36] W.D. Stevenson, Z. Ahmed, X.B. Zeng, C. Welch, G. Ungar, and G.H. Mehl, "Molecular organization in the twist-bend nematic phase by resonant X-ray scattering at the Se Kedge and by SAXS, WAXS and GIXRD” Phys. Chem. Chem. Phys., 19, 13449 (2017).

[37] M. Salamończyk, N. Vaupotič, D. Pociecha, C. Wang, C. Zhu, and E. Gorecka, "Structure of nanoscale-pitch helical phases: Blue phase and twist-bend nematic phase resolved by resonant soft X-ray scattering” Soft Matter, 13, 6694 (2017).

[38] E.I. Kats and V.V. Lebedev, "Landau Theory for Helical Nematic Phases" JETP Lett., 100, 110 (2014).

[39] G. Pająk, L. Longa, and A. Chrzanowska, "Nematic twist-bend phase in an external field" Proc. Natl. Acad. Sci., 2018, 115, E10303. b) L. Longa and W. Tomczyk, “Twist-bend nematic phase in the presence of molecular chirality" Liq. Cryst., 45, 2074 (2018).

[40] M.A. Osipov and G. Pająk, "Polar interactions between bent-core molecules as a stabilising factor for inhomogeneous nematic phases with spontaneous bend deformations" Liq. Cryst., 44, 58 (2017).

[41] G. Barbero and I. Lelidis, "Fourth order nematic elasticity and modulated nematic phases: a poor man's approach" Liq. Cryst., 46, 535 (2019).

[42] C. Meyer, I. Dozov, "Local distortion energy and coarse-grained elasticity of the twistbend nematic phase" Soft Matter, 12, 574 (2016).

[43] S. Garoff and R. B. Meyer, "Electroclinic Effect at the A - C Phase Change in a Chiral Smectic Liquid Crystal” Phys Rev. Lett., 38, 848 (1977).

[44] S.X. Wang, J.A.J. Fells, W.C. Yip, T. Ali, J. Lin, C. Welch, G.H. Mehl, M.J. Booth, T.D. Wilkinson, S.M. Morris, S.J. Elston, "Fast and low loss flexoelectro-optic liquid crystal phase modulator with a chiral nematic reflector" Scientific Reports (Nature Publisher Group), 9, 7016 (2019). 
[45] J.A.J. Fells, C. Welch, W.C. Yip, S.J. Elston, M.J. Booth, G.H. Mehl, T.D. Wilkinson, S.M. Morris," Dynamic response of large tilt-angle flexoelectro-optic liquid crystal modulators “ Optics Express, 27 (11), 15184 (2019).

[46] J.A.J. Fells, X. Wang, S.J. Elston, C. Welch, G.H. Mehl, M.J. Booth, S.M. Morris, "Flexoelectro-optic liquid crystal analog phase-only modulator with a $2 \pi$ range and 1 kHz switching” Optics lett., 43 (18), 4362 (2018).

[ 47 ] S. M. Shamid, S. Dhakal, and J.V. Selinger, "Statistical mechanics of bend flexoelectricity and the twist-bend phase in bent-core liquid crystals" Phys. Rev. E, 87, 052503 (2013).

[48] N. Vaupotič, M. Čepič, M.A. Osipov, and E. Gorecka, "Flexoelectricity in chiral nematic liquid crystals as a driving mechanism for the twist-bend and splay-bend modulated phases" Phys. Rev. E, 89, 030501(R) (2014).

[49] J.S. Patel and R.B. Meyer, "Flexoelectric electro-optics of a cholesteric liquid crystal" Phys. Rev. Lett., , 58, 1538 (1987).

[50] V.P. Panov, J.K. Vij, and G.H. Mehl, "Twist-bend nematic phase in cyanobiphenyls and difluoro-terphenyls bimosogens" Liq. Cryst., 44, 147 (2017).

[51] K. Krzyżewska, T. Jaroch, A. Maranda-Niedbała, D. Pociecha, E. Górecka, Z. Ahmed, C. Welch, G. H Mehl, A. Proń, R. Nowakowski, "Supramolecular organization of liquidcrystal dimers - bis-cyanobiphenyl alkanes on HOPG by scanning tunneling microscopy" Nanoscale, 10,16201 (2018).

[52] R.J. Mandle and J.W. Goodby, "Intercalated soft-crystalline mesophase exhibited by an unsymmetrical twist-bend nematogen”, Cryst. Eng. Comm., 18, 8794 (2016).

[53] J. Carvalho, C. Cruz, J. L. Figueirinhas, M. G. Tamba, A. Kohlmeier, G. H. Mehl, "Proton and Deuterium NMR study of the CBC9CB Dimer System" J. Phys. Chem. B, (2019), DOI: 10.1021/acs.jpcb.8b11526.

[54] A. Aluculesei, H. Cachitas, J. Carvalho, F. V. Chavez, J. L. Figueirinhas, P. J. Sebastião, C. Cruz, M. G. Tamba, A. Kohlemeir, G. H. Mehl, Phys. Chem. Chem. Phys., 21, 4523 (2019).

[55] I. Miglioli, C. Bacchiocchi, A. Arcioni, A. Kohlmeier, G.H. Mehl, and C. Zannoni, "Director configuration in the twist-bend nematic phase of CB11CB" J. Mater. Chem. C, 4 (41), 9887 (2016). 
[56] K. Merkel, A. Kocot, J.K. Vij. "Orientational order and dynamics of the dendritic liquid crystal organo-siloxane tetrapodes determined using dielectric spectroscopy" Phys. Rev. E, 73, 051702 (2006).

[57] H. Toriyama, S. Sugimari, K. Moriya, D. A. Dunmur, and R. Hanson, "Dielectric Study of Dipole-Dipole Interactions in Anisotropic Solutions" J. Phys. Chem., 100, 307. (1996).

[58] W.T. Coffey and Y.P. Kalmykov, "Rotational Diffusion and Dielectric Relaxation in Nematic Liquid Crystals" Adv. Chem. Phys., Editors: I. Prigogine, S.A. Rice, J.K. Vij 113, 427 (2000).

[59] M. Stocchero, A. Ferrarini, G.J. Moro, D.A. Dunmur, G.R. Luckhurst "Molecular theory of dielectric relaxation in nematic dimers. J. Chem. Phys. 121 (16), 8079 (2004).

[60] N. Sebastiána, B. Robles-Hernández, S. Diez-Berart, J. Salud, G.R. Luckhurst, D.A. Dunmur, D.O. López, M.R. de la Fuente. "Distinctive dielectric properties of nematic liquid crystal dimers" Liq. Cryst., 44 (1), 177 (2017).

[61] A. Vanakaras and D. Photinos, "A molecular theory of nematic-nematic phase transitions in mesogenic dimers" Soft Matter, 12, 2208 (2016).

[62] R. J. Mandle, E. J. Davis, C. T. Archbold, S. J. Cowling and J. W. Goodby, "Microscopy studies of the nematic $\mathrm{N}_{\mathrm{TB}}$ phase of1,11-di-(100-cyanobiphenyl-4-yl)undecane" $J$. Mater. Chem. C, 2, 556, (2014).

[63] a) G.K. Youngren and A. Acrivos, "Rotational friction coefficients for ellipsoids and chemical molecules with the slip boundary condition" J. Chem. Phys., 63, 3846 (1975). b) R.J. Sension and R.M. Hochstrasser, "Comment on: Rotational frictioncoefficients for ellipsoids and chemicalmolecules with slip boundary conditions" J. Chem. Phys., 98, 2490 (1993).

[64] C. Meyer, I. Dozov, P. Davidson, G.R. Luckhurst, I. Dokli, A. Knezevic, A. Lesac, "Electric-field effects in the twist-bend nematic phase" Proc. SPIE 10555, Emerging Liquid Crystal Technologies XIII, 105550Z (8 February 2018); doi: $10.1117 / 12.2301296$.

[65] S. Stojadinovic, A. Adorjan, and S. Sprunt, H. Sawade, A. Jakli, "Dynamics of the nematic phase of a bent-core liquid crystal”, Phys. Rev. E 66, 060701 R (2002).

[66] G. Shanker, M. Nagaraj, A. Kocot, J. к. Vij, M. Prehm, C. Tschierske, "Nematic Phases in 1,2,4-Oxadiazole Based Bent-Core Liquid Crystals - Is There a Ferroelectric Switching?", Adv. Funct. Mater., 22, 1671-1683 (2012). 
[67] D. R. Link, G. Natale, R. Shao, J.E. Maclennan, N.A. Clark, E. Korblova, D.M. Walba, "Spontaneous Formation of Macroscopic Chiral Domains in a Fluid Smectic Phase of Achiral Molecules", Science, 278, 1924 (1997).

[68] D. Pociecha, C.A. Crawford, D.A. Paterson, J.M.D. Storey, C.T. Imrie, N. Vaupotic, E. Gorecka, "Critical behavior of the optical birefringence at the nematic to twist-bend nematic phase transition, Phys. Rev. E, 98, 052706 (2018). 